\title{
Assessment of Patient Perspectives and Barriers to Self- Infusion of Augmentation Therapy for Alpha-1 Antitrypsin Deficiency During the COVID-19 Pandemic
}

Jacob Colello $\cdot$ Anna Ptasinski $\cdot$ Xiang Zhan · Sundeep Kaur •

Timothy Craig (D)

Received: October 20, 2021 / Accepted: January 6, 2022 / Published online: January 24, 2022

(C) The Author(s) 2022

\section{ABSTRACT}

Introduction: Alpha-1 antitrypsin (AAT) deficiency is an autosomal co-dominant genetic condition that predisposes individuals to pulmonary and hepatic disease, and in severe cases is treated with augmentation by intravenous infusion. Our aim was to assess patient reluctance to transition to self-administered augmentation of alpha-1-antitrypsin, during the pandemic of SARS-CoV-2.

Methods: A phone questionnaire was administered to 22 patients with severe alpha-1-antitrypsin deficiency who were currently receiving AAT augmentation therapy. Inclusion criteria included patients $\geq 18$ years old, diagnosed with AATD, and receiving intravenous AAT protein augmentation therapy. Information was gathered regarding demographics, perspectives on transitioning to self-administered treatment, and anxiety and depression prevalence. Results

Supplementary Information The online version contains supplementary material available at https:// doi.org/10.1007/s41030-022-00182-z.

J. Colello $\cdot$ A. Ptasinski $\cdot$ X. Zhan $\cdot$ S. Kaur

Penn State College of Medicine, Hershey, PA, USA

T. Craig ( $($ )

Division of Pulmonary, Allergy and Critical Care, Department of Medicine and Pediatrics, Penn State Health, 500 University Drive, Hershey, PA 17033, USA

e-mail: tcraig@pennstatehealth.psu.edu were collected anonymously using REDCap. Joint and marginal statistical analysis was done to quantify links between participants' willingness to transition to self-infusion and correlations with sex, age, years of therapy, anxiety, and depression.

Results: Of 22 patients, 14 were male and eight were female. Ages ranged from 36 to 79 years, with an average of 62.5. Genotypes were $\mathrm{ZZ}$ (14), MZ (3), and SZ (2) among others. Average length of intravenous augmentation was 9.5 years. The majority, 16 participants, were aware self-infusion was an option. Eight participants were willing to consider transitioning to self-infusion if trained and educated. Eight patients reported that fear of COVID-19 transmission influenced their decision-making. Above-normal anxiety, and depression scores, were found in four, and six patients, respectively. Neither sex, age, years of treatment, anxiety, or depression were found to be associated with willingness to consider self-infusion therapy.

Conclusions: Although there are many reasons AATD patients may benefit from AAT self-infusion, including decreased exposure to SARS$\mathrm{CoV}-2$, the majority preferred home nurse-infused therapy.

Keywords: Alpha-1-antitrpysin; Anti-1antitrypsin deficiency; COVID-19; SARS-CoV-2; 
COPD; Augmentation therapy; Survey; Home therapy; AATD

\section{Key Summary Points}

Despite the COVID pandemic, patients receiving protein augmentation for Alpha1 antitrypsin deficiency (AATD) are:

1. Satisfied with home nursing care, despite the increased risk of disease transmission.

2. Feel that home nursing care provided during infusion adds significantly to their health care.

3. That concerns of adverse effects and lack of skill were barriers that patients perceived limiting self-infusion.

\section{INTRODUCTION}

Alpha-1 antitrypsin deficiency (AATD) is a disease characterized by genetic loss of functionally sufficient serum levels of alpha-1 antitrypsin (AAT), a strong inhibitor of neutrophil elastase [1, 2]. Without proper protease/ antiprotease balance, uninhibited elastase results in proteolytic destruction of lung parenchyma [3].

The goal of treatment for patients with AATD is to combat the progression of emphysema. The most specific therapy for AATD is plasmapurified-AAT replacement (augmentation) $[4,5]$. This intravenous infusion, given once per week, aims to increase the serum AAT level above the protective threshold [6-8]. Alpha-1 antitrypsin augmentation therapy requires lifelong treatment for individuals in which it is indicated. Commitment to treatment poses a significant burden on patients. The cost of treatment can range from $\$ 60,000$ to $\$ 150,000$ per year depending on drug pricing and nursing care $[2,9]$. In addition, weekly infusions can significantly interrupt a patient and their family's daily activities and lifestyle choices, compounding anxiety. One of the most recent stressors for AATD patients is that their condition places them at extremely high risk of poor outcomes associated with SARS-CoV-2 infection (COVID-19) [10-13].

It is known that healthcare workers are responsible for patient outbreaks of COVID-19 in hospitals and in long-term care facilities $[14,15]$. Due to recurrent exposure to new patients and asymptomatic COVID-19 infection, there is potential for unintentional spread of the virus from health care agency workers who travel to private homes $[16,17]$.

The pandemic has stimulated efforts to make self-administration of therapies more widely available. Several therapies, specifically subcutaneous biologics used to treat severe asthma, have been successfully transitioned to in-home use during the pandemic $[18,19]$. In addition, self-administration of IV therapies has shown to be safe, effective, and to improve quality of life associated with other hereditary diseases, such as hereditary angioedema and hemophilia $[20,21]$. The advantages of self-infusion include allowing patients to manage their own disease, decreasing need for $\mathrm{RN}$ home visits or travel to outpatient infusion centers, allowing freedom to travel, improving quality of life and self-determination, and most importantly, reducing contacts with health care providers and patients to reduce transmission of SARS-CoV-2. It is our assessment that despite these benefits, a very small demand for self-administration amongst AATD patients exists. Studies attempting to characterize this trend, such as evaluating patient perspectives of home therapy and selfinfusion, are limited.

The goal of this study is to understand the barriers related to patients resisting transition from traditional intravenous alpha-1-antitrypsin protein therapy to administration through self-infusion during the COVID-19 pandemic. In this protocol, we did not alter therapy in any way, but instead assessed why patients may or may not be open to converting to self-augmentation, focusing on the pandemic. 


\section{METHODS}

The study protocol was approved on July 3, 2020 by the Human Subjects Protection Office of Pennsylvania State University Institutional Review Board before study initiation. The study was carried out in accordance with the principles embodied in the Helsinki Declaration of 1995 (as revised in 2013). All participants provided verbal informed consent before the survey was conducted. Potential patients were identified using an internal information services database search of patients with diagnostic coding for alpha-1 antitrypsin deficiency (ICD 10- E88.01, ICD 9- 273.4). Inclusion criteria limited participants to those patients $\geq$ 18 years old, diagnosed with severe AATD, and currently receiving intravenous AAT protein augmentation therapy at the time of survey administration. The questionnaire included basic demographic information such as age, gender, and BMI, in addition to disease-specific parameters including genotype, subjective disease severity, current mode of therapy, duration of therapy, preferred drug composition, and opinions on willingness to transition to selfinfusion. The survey can be found in the Supplementary Material. Patients also completed the Beck Anxiety Inventory (BAI) [22] and Beck Depression Inventory (BDI) [23] to evaluate the presence of mood symptoms that may or may not contribute to self-infusion opinions. Study data were collected and managed using REDCap electronic data capture (Nashville, TN, USA) [24]. Descriptive and statistical analyses were performed for the total patient population and for several demographic subgroups: age, gender, years of therapy, BAI score, and BDI score. Survey response data were analyzed using joint association and marginal association. Association between participants' outcome of "willingness to self-infuse" (yes or no) was compared against the predictors of age, sex, years of therapy, BAI and BDI score. In total, 22 patient surveys were considered for analysis; the two patients currently receiving self-infusion were considered to have a response of "yes" for "willingness to self-infuse".

\section{RESULTS}

Query of the internal electronic medical record generated 302 patients with codes for alpha-1 antitrypsin deficiency. A subset of 68 patients was disqualified on the bases of age criteria. Forty-four patients were found to be currently receiving intravenous AAT augmentation upon chart review, 22 of which were successfully contacted and participated in the survey. Of the total sample, 14 of the patients were male, eight were female. Ages ranged from 36 to 79 years, with an average age of 62.5 years old. Of those that participated, the most common genotype was $\mathrm{ZZ}(n=14)$, followed by MZ $(n=3)$, SZ $(n=2)$, null/null $(n=1), \mathrm{F} /$ null $(n=1)$, and Z/null $(n=1)$. Participant demographics are shown in Table 1.

Average length of intravenous augmentation therapy was 9.5 years. In regards to drug composition preference, ten $(45 \%)$ patients preferred pre-mixed liquid, seven $(32 \%)$ had no preference, and five (23\%) preferred freeze-dried composition. The majority, or $16(73 \%)$, of the patients were aware that self-infusion was available to them. In total, eight (40\%) participants were willing to consider transitioning to self-infusion if training and education were provided. Fourteen $(60.0 \%)$ patients were not willing to consider transitioning to self-infusion. There were two (9\%) patients interviewed that currently self-administer their therapy.

Of the predicted reasons for unwillingness to self-infuse, "fear of consequence if improperly injected" (59\%), "Lack of skills (dexterity required for self-administration)" (59\%), and "worry about financial restraints" (36\%), were the most numerous selected. Additional selfreported reasons included perceived trouble managing infusion supplies, and poor venous access. Only eight $(36 \%)$ patients reported that fear of COVID-19 infection was considered when responding to self-infusion willingness question. Patients also cited benefits of current nurse-infused therapy regimens that impeded their willingness to transition to self-infusion. Of note, responses included "comfortability with nursing care and procedures", "appreciation for weekly check-ins and vital sign 
Table 1 Demographics of participants including total participants, sex, age, mean body mass index (BMI), and disease genotype

Total participants

Sex

Male 14

Female

8

Age

$\leq 39$

40-49

50-59

60-69

$70+$

Mean

BMI, mean

Disease genotype

ZZ

MZ

SZ

Z/null

Null/null

F/null

Data are represented as total number of patients who qualified for each category unless otherwise depicted as mean

monitoring", and perceived "continuity of care" through weekly visits. These data are presented in Table 2.

Beck Anxiety Inventory [22] responses demonstrated 18 (82\%) patients with low anxiety scores, which is considered normal, four $(18 \%)$ with moderate anxiety scores and 0 with high anxiety scores. Beck Depression Inventory [23] results found five (23\%) patients with mild depression scores and one $(0.05 \%)$ patient with a moderate depression score. All other participants' (73\%) BDI scores were indicative of normal scoring.
Table 2 Survey responses regarding patient surrounding willingness to transition to self-infusion therapy regimen

Total years of therapy, mean

Awareness of SI option

Yes

No

Willing to transition to SI

Yes

No

12

Currently on SI

Reasons for unwillingness

Fear of injection

Interference with daily activities

1

Financial constraints

8

Fear of consequence if improperly injected

Lack of skills

Fear of infection

3

Memory difficulties

2

Anxiety and depression

Drug preparation

COVID-19 considered

Yes

8

No

BAI Score

Low

Moderate

Severe

BDI Score

Normal

Mild 5

Moderate 1

Severe

0

Drug composition preference

Pre-mixed 10

Freeze dried 
Table 2 continued

\begin{tabular}{l}
\hline No preference \\
\hline $\begin{array}{l}\text { Data are represented as total number of participants who } \\
\text { selected or scored each option, unless otherwise depicted as }\end{array}$ \\
mean \\
SI self-infusion, BAI Beck Anxiety Inventory, BDI Beck \\
Depression Inventory
\end{tabular}

Joint association revealed no statistical significance of survey data. Individual marginal association analysis did not reveal any statistically significant associations. Table 3 presents study data comparing individuals willing to transition to self-infusion and those unwilling to consider transition to self-infusion.

\section{DISCUSSION}

Currently, the only disease-modifying treatment that exists is lifelong AAT augmentation therapy $[25,26]$. Implementation of this treatment usually requires weekly home visits from nursing staff or visits to outpatient infusion clinics-a process that places significant burdens on patients and their families. To alleviate these burdens, users of other IV therapies, such as hereditary angioedema and hemophilia, have successfully transitioned to self-infusion regimens $[27,28]$. In a recent review, Herth and colleagues outline the practical considerations of self-infusion for AATD patients, in light of the COVID-19 pandemic [29]. They assert that the pandemic has offered an opportune time to expand self-administration of AAT, which can improve safety for such at-risk patients [29].

To date, literature dedicated to highlighting patient perspectives surrounding nurse administered home infusion and/or self-infusion is scarce. In a recent study, 16 AATD patients were enrolled in a nurse-administered home infusion program to reduce potential COVID-19 transmission [30]. Subsequent follow-up of patient perspectives revealed significant quality of life improvement and decreased stress when compared to traveling to a hospital for an infusion [30]. In addition, a large survey of 555 patents by AlphaNet Disease Management and
Table 3 Survey responses stratified comparing individuals willing to transition to self-infusion and those unwilling to consider transition to self-infusion

\begin{tabular}{lll}
\hline Willingness to transition to SI & Yes & No \\
\hline Age & & \\
$\leq 39$ & 1 & 0 \\
$40-49$ & 3 & 0 \\
$50-59$ & 2 & 2 \\
$60-69$ & 1 & 6 \\
$70+$ & 3 & 4
\end{tabular}

Disease genotype

$\mathrm{ZZ}$

MZ

$\begin{array}{ll}6 & 8 \\ 2 & 1 \\ 0 & 2 \\ 0 & 1 \\ 1 & 0 \\ 1 & 0\end{array}$

F/null

Awareness of SI

Yes

$6 \quad 10$

No

42

COVID-19 considered

Yes

$4 \quad 4$

No

6

8

BAI

Normal 7

11

Moderate

3

1

BDI

Normal 9

Mild/moderate

Data are represented as total number of participants who selected or scored each option

$S I$ self-infusion, $B A I$ Beck Anxiety Inventory, $B D I$ Beck Depression Inventory

Prevention Program attempted to gather insight into patient perspectives regarding AAT selfadministration prior to the COVID-19 
pandemic [31]. It was found that only a small subset $(9.2 \%)$ had ever self-administered [31]. Despite their small sample, all patients were very satisfied or satisfied with their self-infusion regimen and were trained efficiency in only 2-3 supervised sessions [31]. Of those who did not self-administer, the majority of patients cited satisfaction with their current regimen or lack of confidence as barriers. Researchers concluded that efforts to expand clinician and patient education programs would increase awareness and confidence of self-administration [31]. In light of the present COVID-19 pandemic, we attempted to further understand patient opinions surrounding nurse administered home therapy vs. self-infusion and hypothesized that patients would be more willing to consider transitioning to self-infusion to decrease risk of COVID-19 transmission from home nurses who may be treating several patients.

In our survey, we found that a majority of patients currently on nurse-administered infusions would prefer to continue their current mode of therapy. Survey participants expressed overall satisfaction with their current regimen, citing confidence in nursing proficiency to achieve venous access with minimal attempts, which was contrary to their anticipated skill level if attempted alone. This is consistent with AlphaNet's findings in the aforementioned survey [31]. The majority of patients have longstanding relationships with the same nurse over many years, in which they are able to build trust and gain confidence in. In fact, the lack of trusted nurses had caused at least two survey participants to transition to outpatient infusion clinics. In addition, many patients who were not open to self-administration at the time of survey, admitted they had considered self-infusion in the past when there were periods of inconsistency of nursing staff. Only one patient reported that infusions were interrupted due to COVID-19-related logistical issues. A new insight gained from the study was that the largest number of patients appreciate the additional care and health maintenance associated with in-home nursing. Nursing staff routinely performs basic health assessments before, during, and after AAT infusion including documentation of blood pressure, temperature, respiratory rate, cardiac and lung auscultation findings, as well as the presence of any new symptoms. Such supplemental assessment and continuity of care cannot be matched by selfinfusion regimen alone. We believe these perspectives highlight an overarching theme in patient care, in that a streamlined process, with limited pain and complications can outweigh other advantages such as self-determination.

The burden of chronic disease, including AATD, has been associated with increased anxiety and depression [9, 32]. For patients with AATD, anxiety and depression can contribute to worsening quality of life and dyspnea. Previous analysis of anxiety in AATD patients using the Beck Anxiety Inventory found elevated scores in over $9 \%$ of individuals, indicative of higher rates of anxiety [9]. It is hypothesized that multiple risk factors contribute to emotional illness in AATD such as decreased functional status and lifestyle limitations, uncertainty regarding disease progression and exacerbations, lifelong nature and cost of treatment, lack of cure, and genetic risk of family members, among others. The threat of severe illness, such as COVID-19 infection has certainly brought about additional cause for anxiety including risk of death or disease, death of family members and friends, social isolation, and financial insecurity. In this survey, we attempted to assess patient anxiety and depression using BAI and BDI tools. We postulated that patients with increased assessment scores would be less likely to be willing to transition to self-infusion. Unfortunately, insufficient sample size made these associations unable to be established.

As discussed previously, AATD patients are at increased risk of poor outcomes associated with COVID-19 infection [10-13]. In light of this, we hypothesized that during the current pandemic, the reduced exposure of self-infusion may outweigh the benefits of current therapy for AATD patients. While the fear of COVID-19 did play a part in patient approaches to protein augmentation, anecdotal analysis did not find that possible increased risk of COVID-19 outweighed the benefits of current therapy for survey participants. Through this survey, we found that AATD patients were aware of their COVID-19 susceptibility due to clinician and nursing 
education. For these reasons, they stated their adherence to necessary safety protocols including limiting social interaction, proper personal protective equipment utilization, sanitization, and health monitoring. Furthermore, survey participants were confident in safety procedures utilized by in-home and in-office nursing staff. Overall, patients' perspectives revealed that increasing accessibility of COVID-19 vaccination signified an impending end to their increased risk of infection. It is evident from our survey that although self-infusion would likely reduce COVID-19 transmission risk, the perceived benefits were not enough to ultimately increase likelihood of transition to self-infusion.

A limitation of this study was that it was restricted to a relatively small subset of patients within a single health system. AATD is a rare disease and only $10 \%$ of patients are diagnosed, and of them only those with severe apha-1-deficiency should receive augmentation therapy. For these reasons, the small sample size can be defended. Second, multiple health care providers prescribed the augmentation, and it was not our task to confirm that prescribers adhered to the ATS criteria for augmentation [8]. It is the author's opinion that augmentation is indicated for severe AATD, which excludes MZs genotypes. Third, only two patients in this survey were currently on self-infusion. Gaining perspectives from a larger sample of patients who are currently using self-infusion methods may reveal a more complete understanding of the barriers AATD patients face. Furthermore, survey participants were not adequately educated on the process, benefits, and drawbacks of self-infusion before answering survey questions. We cannot predict if more education and awareness would have led to increased willingness to consider self-infusion and future studies should determine if education would sway decision on self-infusion.

\section{CONCLUSIONS}

This study has both solidified current understandings of patient perceptions regarding alpha-1 antitrypsin infusion options and brought to light additional considerations for the future, even in the face of a pandemic. Of greatest importance is that all current and future treatment options for AATD should strive to maintain safety and efficacy while being patient centric.

\section{ACKNOWLEDGEMENTS}

We would like to thank the participants of the study.

Funding. No industry funding or sponsorship was received for this study or publication of this article. This project was supported by NIH/NCATS Colorado CTSA Grant Number UL1 TR002535. Its contents are the authors' sole responsibility and do not necessarily represent official NIH views. No Rapid Service Fee was received by the journal for the publication of this article.

Authorship. All named authors meet the International Committee of Medical Journal Editors (ICMJE) criteria for authorship for this article, take responsibility for the integrity of the work as a whole, and have given their approval for this version to be published.

Author Contributions. JC: study design, survey implementation, data input, and manuscript drafting. AP: manuscript drafting and editing. SK: manuscript editing and gathering of literature references. XZ: primary statistician, head of statistical analysis. TC: concept design, supervision, manuscript editing, project administration.

Disclosures. Jacob Colello, Xiang Zhan, and Anna Ptasinski have nothing to disclose. Sundeep Kaur has changed their affiliation since the completion of this study. They are now affiliated with Zucker School of Medicine at Hofstra/ Northwell Residency Program at North Shore University Hospital and Long Island Jewish Medical Center. Timothy Craig speaks and does research for CSL Behring, Grifols and Takeda, and is the Director of Penn State's Clinical Resource Center for the Alpha-1 Foundation. 
Compliance with Ethics Guidelines. The study protocol was approved on July 3, 2020 by the Human Subjects Protection Office of Pennsylvania State University Institutional Review Board before study initiation. The study was carried out in accordance with the principles embodied in the Helsinki Declaration of 1995 (as revised in 2013). All participants provided verbal informed consent before the survey was conducted.

Data Availability. The datasets generated during and/or analyzed during the current study are available from the corresponding author on reasonable request.

Open Access. This article is licensed under a Creative Commons Attribution-NonCommercial 4.0 International License, which permits any non-commercial use, sharing, adaptation, distribution and reproduction in any medium or format, as long as you give appropriate credit to the original author(s) and the source, provide a link to the Creative Commons licence, and indicate if changes were made. The images or other third party material in this article are included in the article's Creative Commons licence, unless indicated otherwise in a credit line to the material. If material is not included in the article's Creative Commons licence and your intended use is not permitted by statutory regulation or exceeds the permitted use, you will need to obtain permission directly from the copyright holder. To view a copy of this licence, visit http://creativecommons.org/licenses/by$\mathrm{nc} / 4.0 /$.

\section{REFERENCES}

1. Strange C. Anti-proteases and alpha-1 antitrypsin augmentation therapy. Respir Care. 2018;63(6): 690-8. https://doi.org/10.4187/respcare.05933.

2. Silverman EK, Sandhaus RA. Alpha1-antitrypsin deficiency. N Engl J Med. 2009;360(26):2749-57. https://doi.org/10.1056/NEJMcp0900449.

3. Petrache I, Hajjar J, Campos M. Safety and efficacy of alpha-1-antitrypsin augmentation therapy in the treatment of patients with alpha-1-antitrypsin deficiency. Biol Targ Ther. 2009;3:193-204.

4. Quinn M, Ellis P, Pye A, Turner AM. Obstacles to early diagnosis and treatment of alpha-1 antitrypsin deficiency: current perspectives. Ther Clin Risk Manag. 2020;16:1243-55. https://doi.org/10.2147/ TCRM.S234377.

5. Traclet J, Delaval P, Terrioux P, Mornex JF. Augmentation therapy of alpha- 1 antitrypsin deficiency associated emphysema. Rev Mal Respir. 2015;32(4): 435-46. https://doi.org/10.1016/j.rmr.2014.10.001.

6. Chapman KR, Burdon JGW, Piitulainen E, et al. Intravenous augmentation treatment and lung density in severe [alpha] 1 antitrypsin deficiency (RAPID): a randomised, double-blind, placebo-controlled trial. Lancet. 2015;386(9991):360-8. https:// doi.org/10.1016/S0140-6736(15)60860-1.

7. Edgar RG, Patel M, Bayliss S, Crossley D, Sapey E, Turner AM. Treatment of lung disease in alpha-1 antitrypsin deficiency: a systematic review. Int J Chron Obstruct Pulmon Dis. 2017;12:1295-308. https://doi.org/10.2147/COPD.S130440.

8. American Thoracic Society/European Respiratory Society Statement. Standards for the diagnosis and management of individuals with alpha-1 antitrypsin deficiency. Am J Respir Crit Care Med. 2003;168(7):818-900. https://doi.org/10.1164/ rccm.168.7.818.

9. Beiko T, Strange C. Anxiety and depression in patients with alpha-1 antitrypsin deficiency: current insights and impact on quality of life. Ther Clin Risk Manag. 2019;15:959-64. https://doi.org/ 10.2147/TCRM.S175369.

10. Zhao Q, Meng M, Kumar R, et al. The impact of COPD and smoking history on the severity of COVID-19: a systemic review and meta-analysis. J Med Virol. https://doi.org/10.1002/jmv.25889

11. Dutta AK, Goswami K. Host genomics of COVID-19: evidence point towards Alpha 1 antitrypsin deficiency as a putative risk factor for higher mortality rate. Med Hypothes. 2021;147:110485. https://doi. org/10.1016/j.mehy.2021.110485.

12. de Loyola MB, dos Reis TTA, de Oliveira GXLM, da Fonseca Palmeira J, Argañaraz GA, Argañaraz ER. Alpha-1-antitrypsin: a possible host protective factor against COVID-19. Rev Med Virol. https://doi. org/10.1002/rmv.2157

13. CDC. COVID-19 and Your Health. Centers for Disease Control and Prevention. Published February 11, 2020. Accessed 19 Feb 2021. https://www.cdc. gov/coronavirus/2019-ncov/need-extraprecautions/people-with-medical-conditions.html 
14. Heinzerling A, Stuckey MJ, Scheuer T, et al. Transmission of COVID-19 to health care personnel during exposures to a hospitalized patient-Solano County, California, February 2020. Morb Mortal Wkly Rep. 2020;69(15):472-6. https://doi.org/10. 15585/mmwr.mm6915e5.

15. McMichael TM, Clark S, Pogosjans S, et al. COVID19 in a long-term care facility-King County, Washington, February 27-March 9, 2020. Morb Mortal Wkly Rep. 2020;69(12):339-42. https://doi. org/10.15585/mmwr.mm6912e1.

16. Kimball A, Hatfield KM, Arons M, et al. Asymptomatic and presymptomatic SARS-CoV-2 infections in residents of a long-term care skilled nursing facility-King County, Washington, March 2020. Morb Mortal Wkly Rep. 2020;69(13):377-81. https://doi.org/10.15585/mmwr.mm6913e1.

17. Rowe TA, Patel M, O'Conor R, McMackin S, Hoak V, Lindquist LA. COVID-19 exposures and infection control among home care agencies. Arch Gerontol Geriatr. 2020;91:104214. https://doi.org/10.1016/j. archger.2020.104214.

18. Lombardi C, Bagnasco D, Passalacqua G. Biological agents for severe asthma: the evolution of the athome self-injection approach. Curr Opin Allergy Clin Immunol. 2020;20(4):421-7. https://doi.org/ 10.1097/ACI.0000000000000656.

19. Timmermann H, Mailänder C. Home self-administration of biologics-a German survey among omalizumab-treated patients with severe asthma and their treating physicians. Pneumol Stuttg Ger. 2020;74(2):103-11. https://doi.org/10.1055/a-1069. 0900 .

20. Longhurst HJ, Carr S, Khair K. C1-inhibitor concentrate home therapy for hereditary angioedema: a viable, effective treatment option. Clin Exp Immunol. 2007;147(1):11-7. https://doi.org/10. 1111/j.1365-2249.2006.03256.x.

21. Schrijvers LH, Beijlevelt-van der Zande M, Peters M, Schuurmans MJ, Fischer K. Learning intravenous infusion in haemophilia: experience from the Netherlands. Haemoph Off J World Fed Hemoph. 2012;18(4):516-20. https://doi.org/10.1111/j.13652516.2012.02752.x.

22. Beck AT, Epstein N, Brown G, Steer RA. An inventory for measuring clinical anxiety: psychometric properties. J Consult Clin Psychol. 1988;56(6): 893-7. https://doi.org/10.1037//0022-006x.56.6. 893.

23. Beck AT, Ward CH, Mendelson M, Mock J, Erbaugh J. An inventory for measuring depression. Arch Gen
Psychiatry. 1961;4:561-71. https://doi.org/10.1001/ archpsyc.1961.01710120031004.

24. Harris PA, Taylor R, Thielke R, Payne J, Gonzalez N, Conde JG. Research electronic data capture (REDCap)-a metadata-driven methodology and workflow process for providing translational research informatics support. J Biomed Inform. 2009;42(2): 377-81. https://doi.org/10.1016/j.jbi.2008.08.010.

25. Stoller JK, Aboussouan LS. A review of [alpha]^sub $1^{\wedge}$-antitrypsin deficiency. Am J Respir Crit Care Med. 2012;185(3):246-59.

26. Wozniak J, Wandtke T, Kopinski P, ChorostowskaWynimko J. Challenges and prospects for alpha-1 antitrypsin deficiency gene therapy. Hum Gene Ther. 2015;26(11):709-18. https://doi.org/10.1089/ hum.2015.044.

27. Bygum A, Andersen KE, Mikkelsen CS. Self-administration of intravenous C1-inhibitor therapy for hereditary angioedema and associated quality of life benefits. Eur J Dermatol EJD. 2009;19(2): 147-51. https://doi.org/10.1684/ejd.2008.0603.

28. Teitel JM, Barnard D, Israels S, Lillicrap D, Poon MC, Sek J. Home management of haemophilia. Haemophilia. 2004;10(2):118-33. https://doi.org/ 10.1046/j.1365-2516.2003.00853.x.

29. Herth FJF, Sandhaus RA, Turner AM, Sucena M, Welte T, Greulich T. Alpha 1 antitrypsin therapy in patients with alpha 1 antitrypsin deficiency: perspectives from a registry study and practical considerations for self-administration during the COVID-19 pandemic. Int J Chron Obstruct Pulmon Dis. 2021;16:2983-96. https://doi.org/10.2147/ COPD.S325211.

30. Annunziata A, Lanza M, Coppola A, Andreozzi P, Spinelli S, Fiorentino G. Alpha-1 antitrypsin deficiency: home therapy. Front Pharmacol. 2021;12: 575402. https://doi.org/10.3389/fphar.2021. 575402 .

31. Sandhaus RA, Boyd BS. Alpha 1 antitrypsin therapy: a satisfaction survey of individuals self-administering. In: A46. TOPICS IN ALPHA-1-ANTITRYPSIN DEFICIENCY. American Thoracic Society International Conference Abstracts. American Thoracic Society; 2018:A1758-A1758. https://doi.org/10. 1164/ajrccm-conference.2018.197.1_ MeetingAbstracts.A1758

32. Kunik ME, Roundy K, Veazey C, et al. Surprisingly high prevalence of anxiety and depression in chronic breathing disorders. Chest. 2005;127(4): 1205-11. https://doi.org/10.1378/chest.127.4.1205. 